\title{
The Nature of RV Tauri Stars
}

\author{
T. Lloyd Evans \\ South African Astronomical Observatory, P. O. Box 9, Observatory \\ 7935, South Africa
}

\begin{abstract}
The infrared colours of the RV Tauri stars fall in a welldefined and thinly-populated region of the IRAS [12] - [25], [25] - [60] diagram. This provides an alternative method of searching for such stars. Stars with these IRAS colours as well as the $K$ and $L$ excesses which are also typical of RV Tauri stars have spectral types from B to M. The characteristic variability of RV Tauri-type has been found for several stars with spectral types typical of known RV Tauri stars, while stars of different spectral types do not vary in this way. RV Tauri stars are the stars with these colours, probably indicative of a dusty circumstellar disk, which lie within the Cepheid instability strip.
\end{abstract}

\section{Introduction}

The RV Tauri stars are defined as radially-pulsating stars with alternating deep and shallow minima. An additional criterion, that they are of late spectral type (Kholopov et al. 1985), is required to avoid possible confusion with eclipsing variables of the $\beta$ Lyrae type which could arise if the light curve was the only observational datum. The Type II Cepheids, or W Virginis stars, in globular clusters begin to show alternating minima at periods longer than about 25 days and a few are recognised as being of RV Tauri type. A similar situation holds for stars in the Large Magellanic Cloud (Pollard et al. 1996; Alcock et al. 1998). The galactic field population includes some RV Tauri stars with stronger metal lines and molecular features than those in globular clusters. Many of these strong-lined stars were found to have very large infrared excesses by Gehrz \& Woolf (1970); later work showed that their colours in both the IRAS [12] - [25], [25] - [60] and the $J-K, K-L$ diagrams distinguish them from the great majority of the red variables which form the bulk of the stars in the IRAS catalogue (Lloyd Evans 1985; Raveendran 1989).

These infrared colours provide a means to discover new examples with selection effects which are quite different from those - primarily optical brightness and large amplitude - imposed by the traditional variable star surveys using blue-sensitive plates. The known RV Tauri stars in the IRAS Point Source Catalogue (Beichman et al. 1988) are largely confined to a rectangle bounded by $[12]-[25]=1.00-1.55,[25]-[60]=0.20-1.00$, which we call the RV Tauri box. Two RV Tauri stars with strong carbon features in their optical spectra (Preston et al. 1963; Lloyd Evans 1974), AC Her and RU Cen, have small near 
infrared excesses but much redder IRAS colours and suggest an extension to $[12]-[25]=2.4,[25]-[60]=1.2$.

\section{Observations}

A list of all the stars with good quality photometry in these two regions in the IRAS Point Source Catalogue was drawn up using a programme provided by $\mathrm{Dr}$ R. M. Catchpole. The positions of over 1000 sources were then examined on the Palomar, ESO and SRC Sky Survey prints and candidate stars were identified; many fields were empty or too crowded while others contained faint red stars which are plausible candidates. Infrared photometry was obtained for 370 stars and spectra were taken for many of them. Most of the stars in the RV Tauri box are $M$ stars, while the extension contains many visually-bright young stars which are less easy to distinguish from the stars which are sought. Stars with typical excesses at $K$ and $L$ are found in all spectral classes from B to M; those of types B and A are Herbig Ae/Be stars and in many cases had already been identified by others. The $M$ stars with infrared excesses are clearly not RV Tauri stars. We consider the 20 stars of types F, G and K as possible RV Tauri stars.

Repeated infrared observations over the period since initial identification (the project started in 1989) and the IRAS estimate of the probability of variability give an indication of the variability of a star, while more intensive observations in the $U B V R I$ passbands were made by the service observers on the $0.5 \mathrm{~m}$ telescope over one or more observing seasons for some of the stars. Several stars are too faint or too crowded for aperture photometry with this telescope, however. Four new RV Tauri stars have been identified in this way, while a known variable, LR Sco $(P=104.4$ days $)$, which was recently identified as a $G$ supergiant (Giridhar et al. 1990, 1992) is also considered to be one. Five stars do not match the photometric properties of RV Tauri stars in one respect or another and are of uncertain status. Many of the others are certainly variable but five stars are either non-variable or have such small amplitudes that they are very unlikely to be RV Tauri stars.

Most of the spectra are consistent with classification as members of group A (Preston et al. 1963), that is they have quite strong metallic lines with $\mathrm{CH}$ and $\mathrm{CN}$ bands which are of normal strength compared to the rest of the spectrum. Two of the four new RV Tauri variables are of the Preston B type which have $\mathrm{CH}$ and $\mathrm{CN}$ bands which are much too strong for the rest of the spectrum; one of them has $\mathrm{C}_{2}$ bands as well. Two stars, one of which is IRAS 20056+1834 which has already been described (Menzies \& Whitelock 1988), have emission in some of the resonance lines of the alkali earths. Both are variable; IRAS 20056+1834 has rather a small amplitude for an RV Tauri star but requires further study, while the other is faint but may have a large amplitude.

\section{Discussion}

The spectral types which have been determined for the IRAS stars observed here and those for a large sample of known RV Tauri stars (Lloyd Evans, in preparation) may be used to define the instability strip for the RV Tauri stars. Different types have been determined for various spectral features by reference 
to a set of standard star spectra, most of which are of supergiants of luminosity class Ib. The spectral type derived from the Balmer series of hydrogen seems most appropriate as a temperature indicator, as the other features are more affected by vagaries of chemical composition and the spectrographic resolution is not adequate to determine the type from the ratios in line pairs. The hydrogen lines are often affected by emission, however, so greatest weight is placed on higher members of the Balmer series. The steep decrement of the emission in these stars makes this a viable proposition. A simple average has been taken for the spectra of each star; this is perhaps more satisfactory for the known RV Tauri stars, which were usually observed more often and for which the already known periods enabled good coverage of the cycle to be obtained.

The known RV Tauri stars fall in the range F5 to G2, with very few earlier than F7. There are a very few stars between G5 and K2; their membership of the group may be questioned. Four of the non-variables among our new sample are earlier than F5, while amplitudes increase from F5 to G3 with a doubtful variable at F8. The late types (G3) for two stars, both confirmed RV Tauri stars, may result from relatively poor phase coverage. One of the five stars noted as possibly not of RV Tauri type from photometric considerations is F5, two are G5 and one, a non-variable, is K5. The spectral types of the new confirmed RV Tauri stars and the three inadequately studied variables are consistent with membership in the class.

The two stars with resonance emission lines are at the tip of the distribution of the $\mathrm{F}, \mathrm{G}, \mathrm{K}$ stars in the $J-K, K-L$ diagram. A similar situation is found for $\mathrm{M}$ and carbon stars with similar $L$ excesses and has been interpreted in terms of a dusty disk which is seen edge-on in the stars with emission as the star is hidden by the disk and can only be seen by reflection from material out of the plane of the disk. T Tauri stars show similar $K$ and $L$ excesses which are attributable to the presence of a disk (eg. Strom et al. 1989). The material which lies above the plane of the disk in these two IRAS stars is the source of the resonance fluorescence emission (Lloyd Evans 1997, following Herbig 1969). It may be concluded that the RV Tauri variables are those members of a class of luminous $\mathrm{F}$ - K stars, probably with low masses as indicated by their kinematics (Joy 1952), which have dusty disks and which pulsate if they lie in the Type II Cepheid instability strip. There is evidence of continuing mass loss from some of these stars (Preston 1964; Pollard et al. 1997) but it seems unrealistic to relate the totality of the dust around them to maintenance by this current activity, as a disk is a stable configuration which will not dissipate as rapidly as an expanding shell.

Lloyd Evans (1985) found that the stars classified on the basis of their light curves as $\mathrm{RVb}$, that is stars with a light variation of much longer period as well as the pulsational variation (Kholopov et al. 1985), were all among the redder stars in the near infrared $J-H, H-K$ and $J-K, K-L$ diagrams. Van Winckel (1999) has explained this correlation as the consequence of an assumed binary nature of the RV Tauri stars, which have circumbinary dust disks. There will be no long-period variation if the system is seen pole-on, whereas the star will be partly obscured when nearest to us in its orbit if the disk is seen from a low angle. The observation that the reddest objects show resonance emission as a 


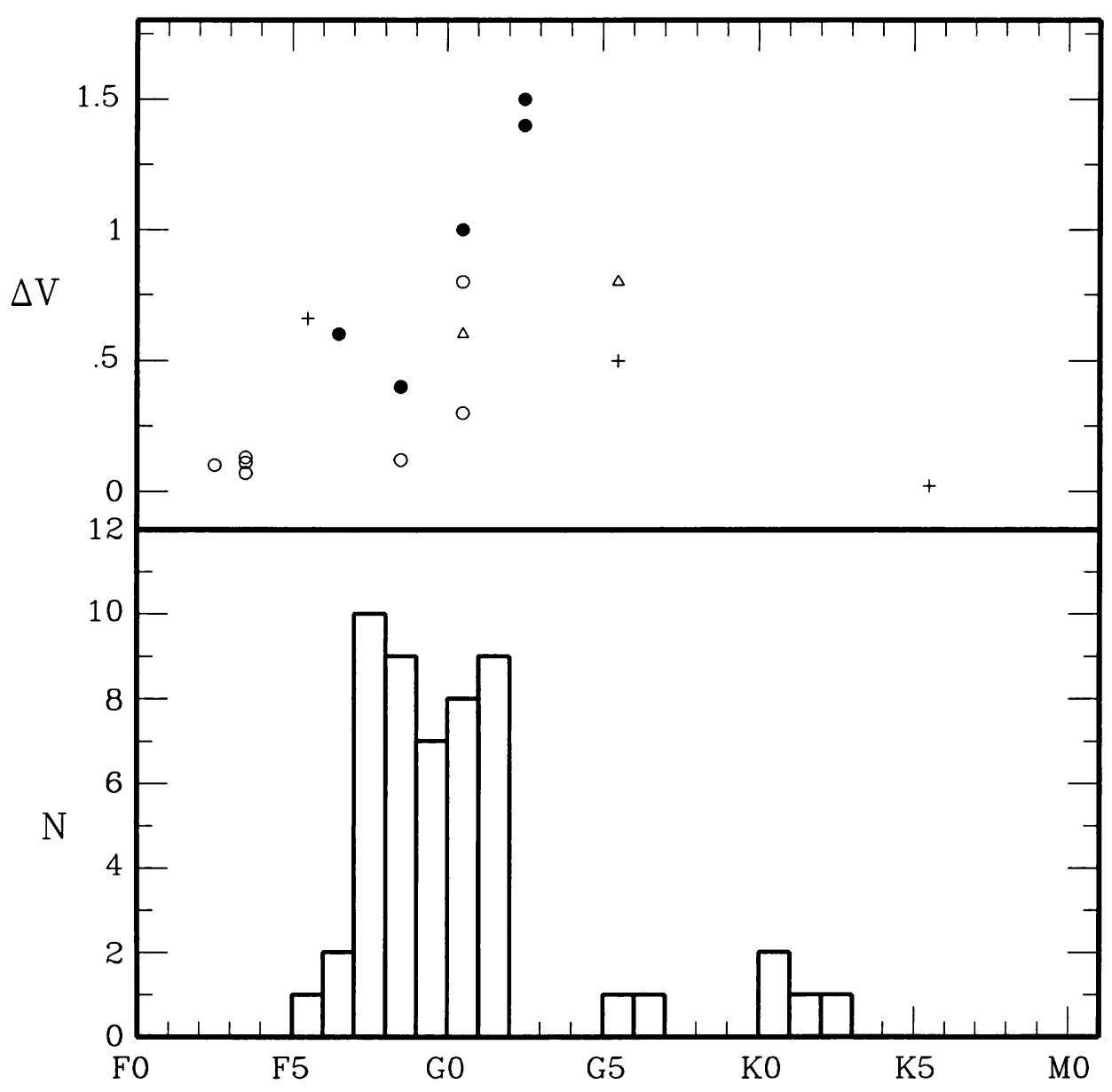

Figure 1. The RV Tauri instability strip. The lower panel shows the number distribution with spectral type of known RV Tauri and related stars, while the upper panel plots the estimated visual amplitude of the candidate RV Tauri stars as a function of spectral type. The symbols are: filled circles, confirmed RV Tauri stars; open circles, stars which are non-variable or of unknown period; open triangles, stars with resonance fluorescence emission; plus signs, stars whose photometric properties differ from those typical of RV Tauri stars. 
result of the star being hidden from direct view confirms the correlation between infrared colour and viewing angle required by Van Winckel's hypothesis.

Acknowledgments. I am most grateful to Dr D. M. Kilkenny who arranged for the $U B V R I$ observations to be made and for supplying the results in a convenient form, and to the $0.5 \mathrm{~m}$ observers, especially Francois van Wyk, who did the observing. I am indebted to Mrs A. A. van der Wielen for assistance in the preparation of this report.

\section{References}

Alcock C., et al., 1998, AJ 115, 1921

Beichman C.A., Neugebauer G., Habing H.J., Clegg P.E., Chester T.J., 1988, Infrared Astronomical Satellite (IRAS) Catalog, NASA, Washington DC Gehrz R.D., Woolf N.J., 1970, ApJ 161, L213

Giridhar S., Rao N.K., Lambert D.L., 1990, Observatory 110, 120

Giridhar S., Rao N.K., Lambert D.L., 1992, JA\&A 13, 307

Herbig G.H., 1969, Contr. Lick Obs. No 302

Joy A.H., 1952, ApJ 115, 25

Kholopov P.N., Samus N.N., Frolov M.S., Goranskij V.P., Gorynya N.A., Kireeva N.N., Kukarkina N.P., Kurochkin N.E., Medvedeva G.I., Perova N.B., Shugarov S.Yu., 1985, General Catalogue of Variable Stars, Nauka Publishing House, Moscow

Lloyd Evans T., 1974, MNRAS 167, 17P

Lloyd Evans T., 1985, MNRAS 217, 493

Lloyd Evans T., 1997, Ap\&SS 251, 239

Menzies J.W., Whitelock P.A., 1988, MNRAS 233, 697

Pollard K.R., et al., 1996, in Variable Stars and the Astrophysical Returns of Microlensing Surveys, 12th IAP Astrophysics Colloquium, R. Ferlet, J.-P.

Maillard and B. Raban (eds.), Edition Frontières, Gif-sur-Yvette, p. 219

Pollard K.R., Cottrell P.L., Albrow M.D., Tobin W., 1997, MNRAS 286, 1

Preston G.W., 1964, ApJ 140, 173

Preston G.W., Krzeminski W., Smak J., Williams J.A., 1963, ApJ 137, 401

Raveendran A.V., 1989, MNRAS 238, 945

Strom K.M., Strom S.E., Edwards S., Cabrit S., Skrutskie M. F., 1989, AJ 97, 1451

Van Winckel H., 1999, this volume, p. 465 


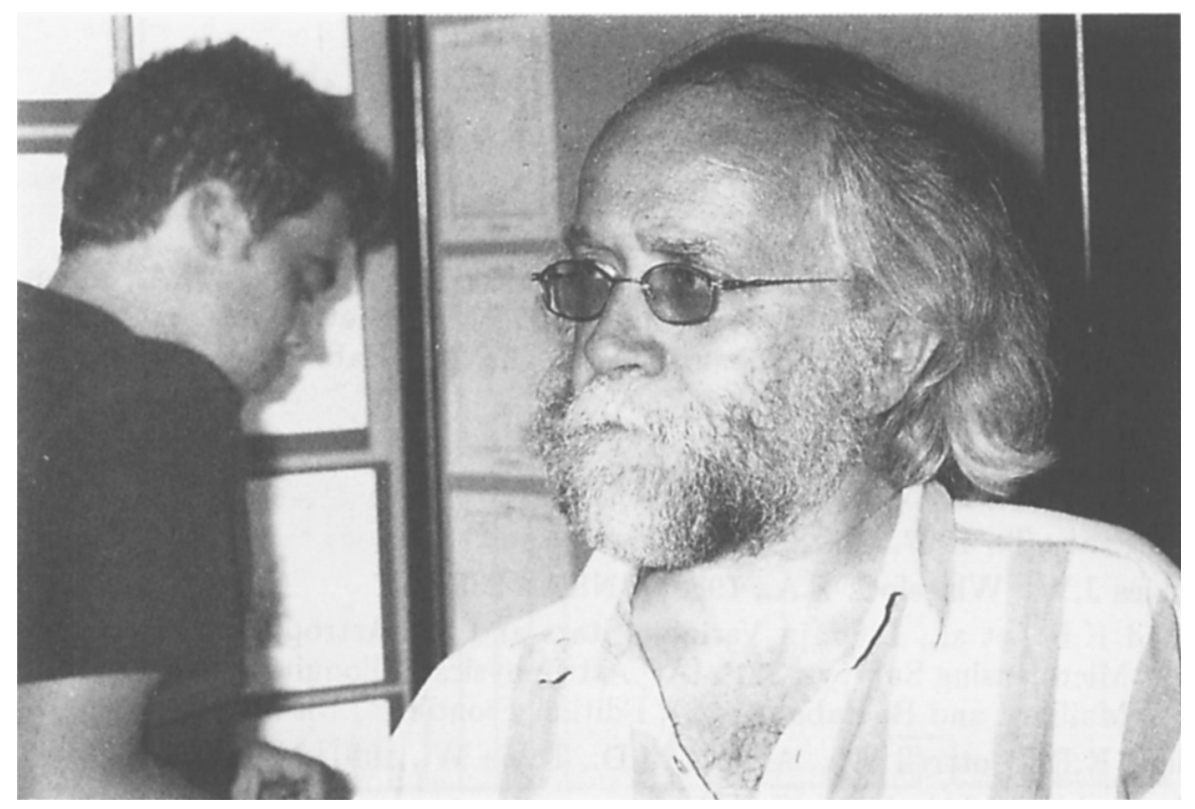

Xander Tielens inspecting the posters 